The American Naturalist, Vol. 142, No. 4. 1993, pp. 604-622.

DOI: $10.1086 / 285560$

http://www.journals.uchicago.edu/loi/an

Copyright is not claimed for this article.

\title{
FISH COHORT DYNAMICS: APPLICATION OF COMPLEMENTARY MODELING APPROACHES
}

\author{
D. L. DeAngelis, ${ }^{*}$ K. A. Rose, ${ }^{*}$ L. B. CROWDER, ${ }^{\dagger}$ E. A. MARSCHALl, ${ }^{\dagger}$ AND D. LiKA ${ }^{\S}$
}

\begin{abstract}
.
The recruitment to the adult stock of a fish population is a function of both environmental conditions and the dynamics of juvenile fish cohorts. These dynamics can be quite complicated and involve the size structure of the cohort. Two types of models, $i$-state distribution models (e.g., partial differential equations) and $i$-state configuration models (computer simulation models following many individuals simultaneously), have been developed to study this type of question. However, these two model types have not to our knowledge previously been compared in detail. Analytical solutions are obtained for three partial differential equation models of early life-history fish cohorts. Equivalent individual-by-individual computer simulation models are also used. These two approaches can produce similar results, which suggests that one may be able to use the approaches interchangeably under many circumstances. Simple uncorrected stochasticity in daily growth is added to the individual-by-individual models, and it is shown that this produces no significant difference from purely deterministic situations. However, when the stochasticity was temporally correlated such that a fish growing faster than the mean $1 \mathrm{~d}$ has a tendency to grow faster than the mean the next day, there can be great differences in the outcomes of the simulations.
\end{abstract}

The great majority of models of ecological populations describe populations as homogeneous collections of organisms. However, to an increasing degree, ecologists have become aware that the internal age and size structures of populations can have a decisive influence on the population dynamics (Ebenman and Persson 1988). Size structure may be particularly important in populations in which growth is fairly plastic and feeding and vulnerability to predation depend on size. The first factor, plasticity in growth, leads to the potential for a wide spectrum of organism sizes in the population, even within cohorts of individuals of the same age. The second factor, size dependence of feeding and vulnerability, means that members of an age-class cohort that have different sizes will have different probabilities of success in surviving and reproducing.

These factors affect the dynamics of a population cohort and thus have many ramifications, both theoretical and practical, in ecology. On the theoretical side, there are questions of appropriate parental strategies (e.g., size of eggs, early parental care) and individual growth strategies in the face of environments with various size-dependent food availabilities and risks of predation. On the practical side, the recruitment of juvenile fish to the adult classes in commercial fish species is extremely important but is not well understood. It is quite possible 
that the dynamics leading to recruitment involve size structure in important ways.

Because of the apparent importance of size structure in the dynamics of many populations, models that consider size structure explicitly have been developed. Models that take into account the states of individual organisms in a population have been called $i$-state models, differentiated from $p$-state models, which have state variables representing the whole population (see Metz and Diekmann 1986). The $i$-states can be any characteristics of individuals - their sizes, ages, or energy reserves, for example. Caswell and John (1992) further classify two types of $i$-state models: distribution models and configuration models. Distribution models assume that a given characteristic that varies in a population forms a continuous distribution, rather like the continuous distribution of velocities of atoms in a gas that is usually assumed in the kinetic theory of gases. Distribution models for size-structured populations are most frequently partial differential equations, such as the McKendrick-von Foerster equation (see, e.g., Sinko and Streifer 1967; Nisbet and Gurney 1982).

The $i$-state configuration models do not assume a continuous distribution of characteristics, such as sizes, but are computer models in which many different individuals, each with its own characteristics, are simulated simultaneously. Thus, the distribution of sizes that occurs in such models is the sum of the simulation of many individuals.

Both $i$-state distribution and $i$-state configuration models have been used extensively to deal with problems involving the size structure of populations. The advantages and disadvantages of each approach have been compared. For example, several of the factors involved in choosing which approach to use have been discussed (DeAngelis and Rose 1992). These factors include the size of the population being modeled, the amount of stochasticity expected in the population, the degree to which the population is well mixed versus stratified, and the complexity of actions by individuals. Large population size, little stochasticity, good mixing, and fairly simple individual behaviors all increase the ease of formulation and accuracy of a partial differential equation model description. Conversely, small population size, high stochasticity, poor mixing, and complex individual behaviors tend to make partial differential equation models poor representations of populations.

Despite such discussions of relative advantages, little or nothing has been done actually to compare in detail the predictions of the two different types of models. In this article we compare precise analytical solutions of partial differential equation models of size-structured populations (one type of $i$-state distribution model) with $i$-state configuration models, in this case Monte Carlo computer simulation models that follow thousands of individuals of a population (the term Monte Carlo refers to the use of pseudorandom number generators in such models to simulate the stochasticity of individuals).

Our article has two basic goals. The first goal is to see just how well the computer simulation configuration models do when they are used to describe problems that can be formulated as analytically solvable partial differential equations. If they do well without one having to use an impractically large number of simulated individuals, this would suggest that the configuration approach and the partial differential equation approach may be validly substituted for each other when it is more convenient to use one or the other. The second goal is to see what happens when we add complexities to these simple population systems that make them less amenable to formulation as partial differential equations but that can be easily handled by means of computer simulations of many individuals. A crucial question is whether some of these complexities, which may reflect what can happen in real populations, have major effects on the dynamics. 
This article deals only with population cohorts, although the results have implications for the more complex problems of the dynamics of total populations and communities.

\section{THE MODELS}

In an attempt to deal with size-structured populations quantitatively, one approach has been to adapt the Leslie matrix approach normally used to describe age-structured populations (Caswell 1989). Instead of probabilities of transition from one age class to another, as in the agestructured Leslie matrix models, in the size-structured models there are probabilities for transition from a given size class to another. Another approach is the McKendrick-von Foerster model, in which size is a continuous variable and the population is described by a partial differential equation for the continuous variable, $f(S, t)$, the density of organisms (number per unit size interval) at size $S$ at a given time (Van Sickle 1977; DeAngelis and Mattice 1980; DeAngelis et al. 1980; Wismer et al. 1985; Metz and Diekmann 1986; DeAngelis and Huston 1987).

The Leslie matrix approach is generally used to follow a whole population on yearly time steps, whereas we will be considering a population cohort during a time period shorter than a year. The relevant analytical model of a cohort is the McKendrick-von Foerster model, and the results of this model will be compared with those of a simulation model.

The formulation of a McKendrick-von Foerster model is described in detail by Metz et al. (1988). To model a single population cohort, one can start with equations that describe the growth and mortality of individuals (called the $i$-model by Metz and his colleagues). A general equation for the growth in size of an individual is

$$
d S / d t=g(S, t)
$$

where $g(S, t)$ is a function of size and time. Mortality can be described by the function $Z(S, t)$ and hatching rate, or the input of new individuals of a given size, $S_{0}$, to the cohort by the function $B\left(S_{0}, t\right)$. Assume that all individuals in the population have the same functions describing their growth rate and mortality. Then the equation for the size distribution function, $f(S, t)$, is

$$
\partial f(S, t) / \partial t+\partial[g(S, t) f(S, t)] / \partial S=-Z(S, t) f(S, t) .
$$

This equation, along with initial conditions on $f(S, 0)$ and/or a function $B\left(S_{0}, t\right)$ describing hatching times of new individuals being added to the cohort through time, is sufficient to describe changes in $f(S, t)$ for the population cohort through time.

This model may be extended to more complex cases in which one or more other parameters vary across the population. For example, suppose that the growth rate of a given fish in the population is described by

$$
g(S, t)=g_{0} S,
$$

where $g_{0}$ is a constant for a given fish but where $g_{0}$ may be different for different individuals. Equation (2) can be solved to find $f_{g}\left(S, g_{0}, t\right)$ for fish with a particular value of $g_{0}$. The total size density function is then found by integrating over all growth rate coefficients in the population: 


$$
f(S, t)=\int_{0}^{\infty} F\left(g_{0}\right) f_{g}\left(S, g_{0}, t\right) d g_{0},
$$

where $F\left(g_{0}\right)$ is the distribution of growth rate coefficients over members of the population.

The compact nature of equation (2) and the availability of methods for solving such equations make the McKendrick-von Foerster model a useful modeling approach for studying the dynamics of population cohorts. However, there are assumptions implicit in equation (2) that question its universal applicability to problems of cohort dynamics. One assumption is that the size density is continuous. This assumption will never be strictly true since individuals in populations are discrete, but it may be a useful approximation for large populations. A second assumption is the deterministic nature of the dynamics. Only one outcome for the development of the model solution through time is possible. In nature, however, one expects that there will be random variations in the mortality and growth of individuals. A third implicit assumption is that correlations among individuals do not exist. For example, one might expect in natural situations that the presence of larger individuals in a population may have some inhibitory effect (e.g., via competition) on smaller individuals and thus affect their performance relative to the case in which there are no larger individuals present. This possibility is ignored in equation (2). For cohorts of larval fish, this should not usually be a serious problem, as there is little evidence of competition among individuals, and larva are often dilute relative to the abundance of their food resource (Hunter 1981).

It is probable that partial differential equation models will fail most often in situations in which populations are relatively small, stochasticity is important, and the potential exists for positive feedbacks to amplify the effects of stochastic events (Huston et al. 1988; Łomnicki 1988). An example might be a cohort of fish or anurans for which food is limiting and in which survival requires that individuals attain a threshold size. In such a case, the great majority of individuals will die. Whether a few survive may depend on stochastic events, such as a few individuals having, by chance, somewhat greater feeding rates and reaching larger sizes. A small, random, higher growth rate by a few individuals can amplify if their larger size gives them a slight competitive advantage. Predicting the outcome of such situations is difficult (Persson 1987; Łomnicki 1988; Wilbur 1988).

The above limitations of the McKendrick-von Foerster model may be overcome by various elaborations of the model. However, these elaborations, such as the addition of stochasticity and interactions among various size classes, add massively to the complexity of this model and defeat much of its advantage of simplicity. In addition, this approach may never be satisfactory for small populations. Because our purpose is to compare the analytical and simulation models, we adopt this simpler model.

A third approach to the handling of size-structured populations is that of Monte Carlo computer simulation. In this approach, initial development of a model is somewhat similar to that of the McKendrick-von Foerster equation. An $i$-model is first developed for individuals in the populations. However, instead of the $i$-state model developing into an equation of the form of equation (2), the Monte Carlo approach attaches an $i$-state model to each member of the population and then follows the individual growth and survival of each member through time. The dynamics of the whole population can be determined by summing over all of the extant individuals at any given time.

This Monte Carlo approach, termed "individual-by-individual," "individual-based," or "individual-oriented" modeling, has many advantages of flexibility (see Huston et al. 1988). 
Because the individual-based approach uses computer simulation, it is relatively simple to make the $i$-state model of each individual stochastic rather than deterministic. Correlation effects between individuals can also easily be included. Small population sizes do not create a problem for the individual-based approach as they do for the McKendrick-von Foerster equation, and in fact they may be an advantage in that each individual is modeled.

The existence of these two approaches should be viewed as an advantage rather than as a problem, since they appear to be complementary. The McKendrick-von Foerster representation should allow analytical solutions for a number of simplified, but nonetheless interesting, situations of cohort dynamics in which the assumptions underlying a partial differential equation model are satisfied. These analytical solutions will allow one to obtain a general understanding of how various parameters affect changes in the cohort through time. The Monte Carlo model allows one to extend the results to more complex cases and to cases, such as that of small population size, in which the partial differential equation approach is not valid.

If these two approaches are indeed complementary, then they should yield approximately the same results in the limit of large population size. This expectation must be tested. The question then arises as to what constitutes a sufficiently large population size for application of the partial differential equation approach. Besides the question of population size, there are also questions of how such things as correlations between fish within the cohort and temporal correlations in fish growth affect dynamics of the cohort.

\section{ANALYSIS OF MODELS}

Three different types of situations for population cohorts were considered, and both $i$ state distribution and $i$-state configuration models were developed. These situations are chosen to be representative of the types of growth that may occur in real populations.

\section{Case 1}

All fish are assumed to have the same initial size (length), $S_{0}$. Growth rate, $g(S, t)$, is a constant, $g_{0}$, independent of length and differs for each fish, being selected from a normal distribution with a mean of $g_{0 m}$ and standard deviation of $b$. Growth is in millimeters per day and does not depend directly on body size. This type of growth pattern is not unusual in larval fishes (Hunter 1981; Houde 1987, 1989; Pepin 1991). The probability of mortality of fish is assumed to vary inversely with length, $Z_{0} / S$, where $Z_{0}$ is a constant. Such an inverse relationship is likely when the mortality is due primarily to predation. Smaller individuals will be more vulnerable to predation and will usually have a greater number of potential predators. Size-dependent mortality is commonly observed in larval fish (Miller et al. 1988).

The $i$-state distribution model, or partial differential equation model, for this situation is

$$
\partial f / \partial t+\partial\left(g_{0} f\right) / \partial S=-\left(Z_{0} / S\right) f
$$

where the growth rates are distributed about $g_{0}$ as

$$
F\left(g_{0}\right)=\left[N_{0} /(2 \pi)^{0.5} b\right] \exp \left[-\left(g_{0}-g_{0 \mathrm{~m}}\right)^{2} / 2 b^{2}\right],
$$


where $N_{0}$ is the total number of fish in the cohort. The analytical solution for this model is

$$
f(S, t)=\left[N_{0} /(2 \pi)^{0.5} b t\right] \exp \left[-\left(S-S_{0}-g_{0 \mathrm{~m}} t\right)^{2} / 2 t^{2} b^{2}\right] \times\left(S_{0} / S\right)^{\left[Z_{0} t /\left(S-S_{0}\right)\right]} .
$$

Case 2

Each member of the population cohort starts at time $t=0$ with a different initial size, $S_{0}$, chosen from a normal distribution with mean, $S_{0 m}$, and standard deviation, $b$. This situation might reasonably occur if eggs produced vary in size across the population. The growth rate, $g$, for each fish of a given size is the same and is a linearly increasing function of size, $g_{l} S$, where $g_{1}$ is a constant. In this case, growth is in percentage of body length per day, which is also a common pattern in larval and juvenile fish (Hunter 1981; Houde 1987). The mortality is assumed to vary inversely with size, $Z_{0} / S$, as in case 1 .

The partial differential equation for this situation is

$$
\partial f / \partial t+\partial\left(g_{1} S f\right) / \partial S=-\left(Z_{0} / S\right) f,
$$

where the initial sizes are distributed as

$$
F\left(S_{0}\right)=\left[N_{0} /(2 \pi)^{0.5} b\right] \exp \left[-\left(S_{0}-S_{0 \mathrm{~m}}\right)^{2} / 2 b^{2}\right] .
$$

The analytical solution for this model is

$$
\begin{aligned}
f(S, t)= & {\left[N_{0} /(2 \pi)^{0.5} b\right] \exp \left[-\left(S e^{-g_{1} t}-S_{0 \mathrm{~m}}\right)^{2} / 2 b^{2}\right] } \\
& \times \exp \left[Z_{0}\left(1-e^{g 1^{1 t}}\right) / g_{1} S\right] \times \exp \left(-g_{1} t\right) .
\end{aligned}
$$

\section{Case 3}

The individuals in the cohort are assumed to hatch at the same size, $S_{0}$, at different times over a spawning season, with the hatching frequency being described by a normal distribution with mean time of hatching, $t_{0}$, and standard deviation, $b$, with the spawning season assumed to extend to three standard deviations on either side of the mean. The growth rate, $g(S, t)$, is assumed to be the same for each fish of a given size and to be described as a logistic function, $g_{I} S\left(1-S / S_{\max }\right)$, so that there is an upper limit on physiological size. This growth function is somewhat more common in models that include juvenile and adult fishes than in larval fishes and only serves here to illustrate the robustness of our comparisons. A constant mortality rate, $\mathrm{Z}_{0}$, is assumed; that is, mortality is assumed to be size-independent. This may be adequate for describing processes of population loss such as transport (Sinclair 1988).

The partial differential equation for this situation is

$$
\partial f / \partial t+\partial\left[g_{1} S\left(1-S / S_{\max }\right) f\right] / \partial S=-Z_{0} f,
$$

with the hatching times assumed to be distributed normally around a mean $t_{0}$ as

$$
B(t)=\left[N_{0} /(2 \pi)^{0.5} b\right] \exp \left[-\left(t-t_{0}\right)^{2} / 2 b^{2}\right] \text { at } S=S_{0} .
$$

Note that we do not prescribe an initial size-frequency distribution $F\left(S_{0}\right)$ here as we did in cases 1 and 2. All members of the cohort are added through the hatching function (12), which 
mathematically is a time-varying boundary condition on size at $S=S_{0}$. The analytical solution for this model is

$$
\begin{aligned}
f(S, t)= & {\left[N_{0} /(2 \pi)^{0.5} b\right]\left[S_{\max } / g_{1}\left(S_{\max }-S\right) S\right] } \\
& \times\left[S_{0}\left(S_{\max }-S\right) / S\left(S_{\max }-S_{0}\right)\right]^{Z_{0} / g 1} \\
& \times \exp \left(-\left\{t-\ln \left[S\left(S_{\max }-S_{0}\right) / S_{0}\left(S_{\max }-S\right)\right] / g_{1}-t_{0}\right\}^{2} / 2 b^{2}\right)
\end{aligned}
$$

The above analytical solutions were evaluated for a sequence of times. In addition to these analytical solutions, equivalent configuration model computer simulations (Rice et al. 1993) were performed of the three model cases by simulating the growth and survival of numerous individuals (200,000 for cases 1 and 2, 20,000 for case 3). A time step of a day was used. On each day each fish grew an increment determined by its growth rate, $g(S, t)$, which in cases 2 and 3 depended on its size, $S$, at the time. Also on each day, whether a given individual survived or not was determined by comparing a pseudorandom number $R$ chosen uniformly on $(0,1)$ with the daily probability of mortality, $Z_{0}$. Thus, mortality was a stochastic event for a given individual. The results of the $i$-state distribution and $i$-state configuration models were then compared.

\section{RESULTS}

\section{Comparisons between Partial Differential Equation Solutions and Simulations}

Case 1.

The comparison between the partial differential equation solution (7) and the Monte Carlo simulation for the size-frequency distribution of the fish population cohort for case 1 is shown in figure 1. The specific values of the parameters used for the comparison were based on growth and mortality for the bloater, Coregonus hoyi (Rice et al. 1987). These values are given in table 1. The cohort size-frequency distributions are shown for three times, $t=20,40$, and 60 d. Agreement between the two models is acceptably close, although the numbers of fish in the Monte Carlo simulation tend to fall slightly below those in the partial differential equation solution, especially for $t=60 \mathrm{~d}$. This is to be expected because the partial differential equation model is a continuous-time model, whereas the Monte Carlo simulation model works on discrete daily time steps. There is a slight difference in continuously compounded loss rates and such losses compounded daily. Over a period of $60 \mathrm{~d}$, such a difference will be visible, as seen in figure 1 . 


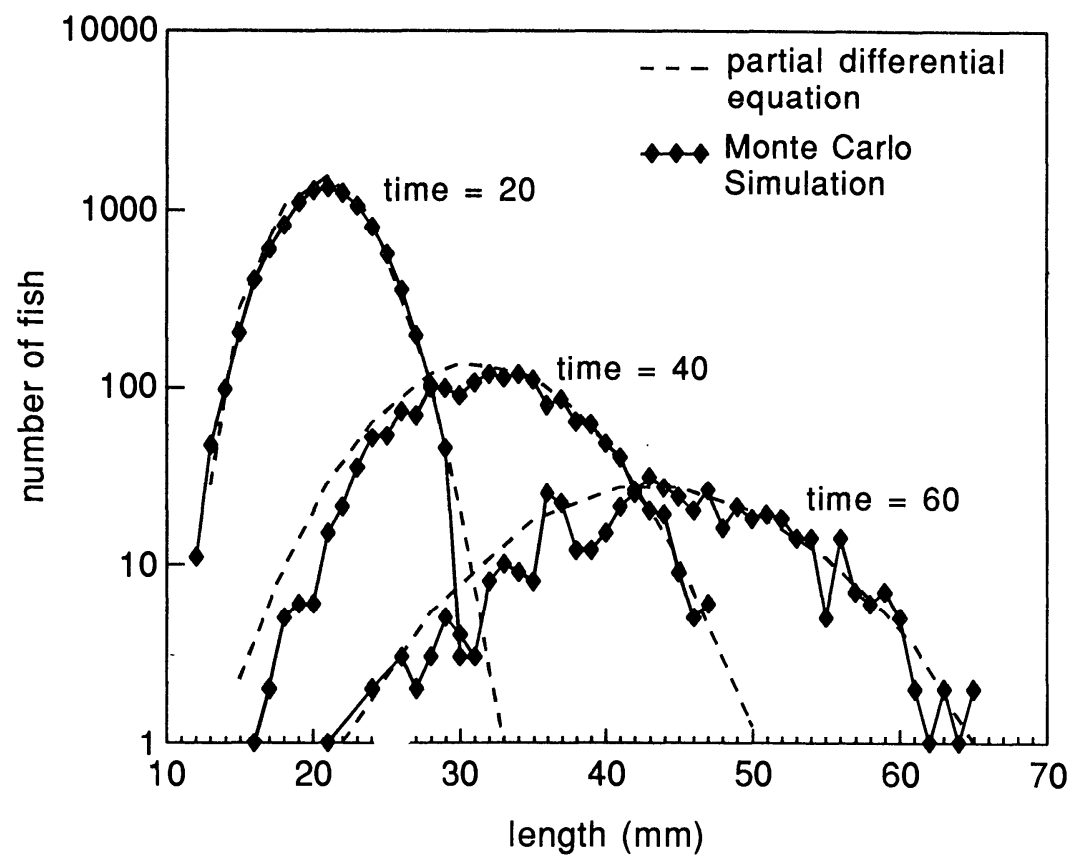

FIG. 1.-Case 1 comparison between the partial differential equation solution, eq. (7) (dashed line), and the equivalent Monte Carlo simulation (solid line) for the size-frequency distribution of a cohort. An initial 200,000 fish were simulated in the Monte Carlo simulation.

The Monte Carlo model gives us the opportunity for modifying the rules for growth arbitrarily in ways that a partial differential equation formulation cannot describe. As a first example, consider the case in which there is some randomness in the daily growth increment due to variability in the daily encounters of individual fish with potential prey. In particular, on each day allow the growth rate of each fish to vary randomly and uniformly within $\pm 0.15 \mathrm{~mm} / \mathrm{d}$ around the mean growth rate of a particular fish. It seems likely that daily growth rates will be correlated, but we first examine the purely stochastic effect, without correlations. The results of this added randomness can be compared with the baseline case of figure 1 in which there is no randomness. The comparison is shown in figure 2 at a time of $60 \mathrm{~d}$, where the open bars represent the numbers in the nonrandom simulation and the solid bars represent the numbers in the random simulation. There is no apparent difference between the two size-frequency distributions. A two-sided Kolmogorov-Smirnov (K-S) test was applied to the distributions, assuming 42 degrees of freedom (for the number of 1-mm size classes). The calculated K-S statistic is 0.0696 . Since the critical value at $\alpha=0.1$ for a two-sided test is 0.05809 and at $\alpha=$ 0.05 is 0.06476 , then $.01<P<.05$, and the two distributions in figure 2 are just barely different at $\alpha=0.05$. Hence, stochasticity by itself seems to have little effect on the average growth rates of individuals.

The small effect of randomness on the size-frequency distribution is not too surprising and can be estimated analytically. Randomness in individual growth of fish could be included in the model (eq. [5]) by adding a second-order term, $0.5 \partial^{2} / \partial S^{2}(D f)$, where $D$ is a diffusion coefficient. In the case in which there is a $\pm 0.15 \mathrm{~mm} / \mathrm{d}$ variability in growth, the associated value of $D$ would be $(0.15)^{2} / 2$, or approximately 0.0112 . Although the full equation (5) cannot be solved analytically when the second-order diffusion term is added, one can estimate the effects of the diffusion term on the size-frequency distribution to show that only an approximate $15 \%$ 
increase in its standard deviation after $60 \mathrm{~d}$ would result. Because in the Monte Carlo simulation the upper limits on the variation in growth were $\pm 0.15 \mathrm{~mm} / \mathrm{d}$, the increase in the standard deviation of the size-frequency distribution should actually be much smaller than the approximate prediction.

TABLE 1

\begin{tabular}{|c|c|c|c|}
\hline & Case 1 & Case 2 & Case 3 \\
\hline$b$ & $.16^{*}$ & $2.0 \dagger$ & $3.0 \ddagger$ \\
\hline$g_{0 \mathrm{~m}}$ & .40 & & \\
\hline$g_{1}$ & & .016 & .03 \\
\hline$N_{0}$ & 200,000 & 200,000 & 20,000 \\
\hline$S_{\max }$ & & $\ldots$ & 50.0 \\
\hline$S_{0}$ & 12.0 & & 12.0 \\
\hline$S_{0 \mathrm{~m}}$ & $\ldots$ & 12.0 & \\
\hline$t_{0}$ & 22 & 20 & 10.0 \\
\hline
\end{tabular}

NOTE.-An ellipsis (...) means that the parameter is not relevant to a particular equation.

* Standard deviation for distribution of growth rates.

$\uparrow$ Standard deviation for distribution of initial sizes.

$\$$ Standard deviation for distribution of hatching times.

It is reasonable to suppose, however, that instead of the purely random daily variations in growth assumed there may be correlations (memory) between the growth increments from day to day. For example, a growth increment on day $t$ that is higher than the mean because of stochasticity is likely to be followed on day $t+1$ by a growth increment that is also higher than the mean. That this can occur in fish is "conventional wisdom" among fishery scientists; it seems likely that a fish that does better than average in feeding on $1 \mathrm{~d}$ will be in good condition the next and thus have better prey capture efficiency.

Studies have been made of the widths of daily rings in otoliths of fish to provide an approximate age/growth history that might confirm this conventional wisdom. It has been shown that wider rings are deposited at higher growth rates and day-to-day ring widths appear to be correlated, but daily increment spacing is difficult to relate precisely to daily growth rates (Rice et al. 1985). Direct measurements of lengths of tagged and multiply recaptured larval fish may be needed to provide proof of day-to-day correlations in growth. However, for present purposes, suppose such correlations exist. To mimic them, we assume that for a given fish the growth increment on day $t$ is chosen as the new mean growth rate. The growth increment for this fish on day $t+1$ is chosen randomly and uniformly over the interval $\pm 0.15 \mathrm{~mm} / \mathrm{d}$ around this new mean. With this rule, in principle the growth increments of some fish could become very large, while others could become very small after a number of days. To prevent growth rates becoming unrealistically large on the one hand or negative on the other, limits of $0.0 \mathrm{~mm} / \mathrm{d}$ and $1.0 \mathrm{~mm} / \mathrm{d}$ (Pepin 1991) were put on the growth rates of individual fish. When these simulations were performed a significant difference was found between the Monte Carlo simulations and the baseline case (fig. 3). The K-S statistic is 0.998, and the two distributions are significantly different at $\alpha=0.01$. Correlated daily growth rates, or memory, caused the distribution of growth rates in the population to broaden, which resulted both in much faster growers and much slower growers. Because the size-dependent mortality acted much more intensely on the slower-growing fish, there was a consequent shifting to the right of the size frequency distribution at $60 \mathrm{~d}$. 


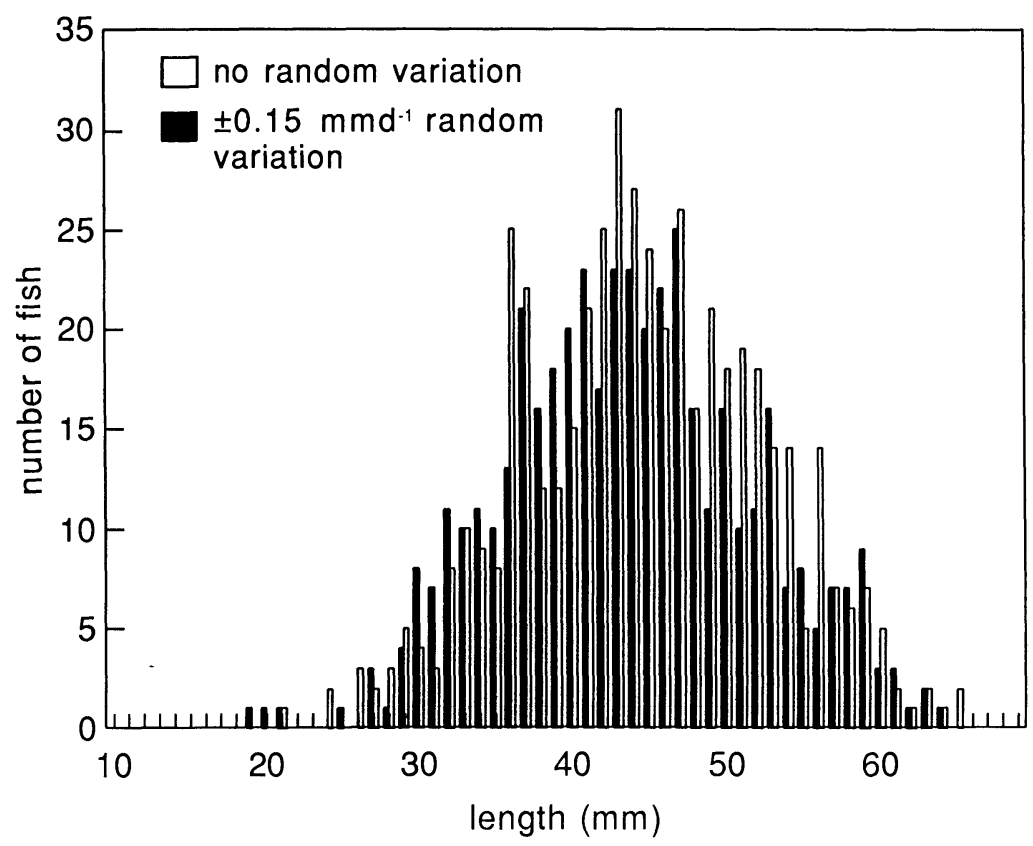

FIG. 2.-Case 1 size-frequency distributions from individual-based computer simulations of a fish cohort after $60 \mathrm{~d}$. The open bars represent the survivors of an initial 200,000 fish, where daily growth of individuals was deterministic (no stochasticity in daily growth). The solid bars represent the equivalent case in which each day there could be a random variation of up to $\pm 0.15 \mathrm{~mm}$ around the mean growth rate.

The choice of $\pm 0.15 \mathrm{~mm} / \mathrm{d}$ as the upper and lower limits on the daily random variation about the mean in growth increment is arbitrary. Actual variances in growth resulting from random effects on feeding could be much greater, so it is worth exploring the effect of larger variance. Simulations indicate that there is a substantial effect of the choice of daily variance on the outcomes of both fraction of survivors and mean length of survivors after $60 \mathrm{~d}$. Figure 4 shows the effect of increasing variance with and without memory. Without memory, there is negligible effect from increased variance. However, with-memory survival increases with increasing variance. This must reflect a larger fraction of fish reaching larger sizes more rapidly and escaping size-dependent mortality. A plot of mean length as a function of increasing variance shows, in the case of correlated daily growth rates, a peculiar dome-shaped response (fig. 5). As the limit on daily variability increases beyond $\pm 0.15 \mathrm{~mm} / \mathrm{d}$, the mean length of survivors at $60 \mathrm{~d}$ starts to decrease. The precise cause is difficult to explain, but it is related to the limitation rule for maximum daily growth that is employed. In figures 4 and 5 the rule puts a limit of $1.0 \mathrm{~mm} / \mathrm{d}$ on the daily growth increment of fish in the simulation. If this limit is increased to $1.5 \mathrm{~mm} / \mathrm{d}$, which is a reasonable large average value for larval fish (Pepin 1991), survival at $60 \mathrm{~d}$ increases to a much larger value (fig. 6), and the mean size after $60 \mathrm{~d}$ reaches a larger peak shifted toward larger values of variance (fig. 7). 


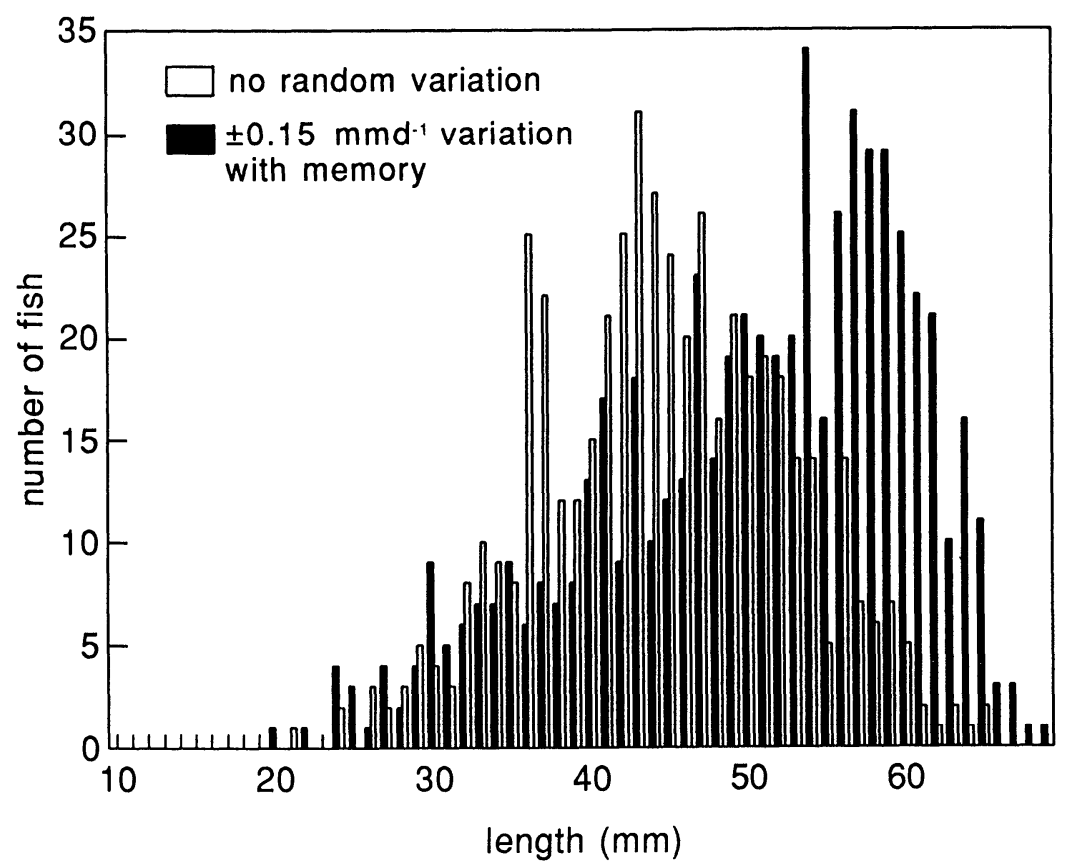

FIG. 3.-Case 1 size-frequency distributions from individual-based computer simulations of a fish cohort after $60 \mathrm{~d}$. The open bars represent the survivors of an initial 200,000 fish, where daily growth of individuals was deterministic. The solid bars represent the equivalent case in which each day there could be a random increment of up to $\pm 0.15 \mathrm{~mm}$ around the preceding day's growth rate. This simulation contrasts with that of fig. 2 because the daily growth is now positively correlated with growth on the preceding day.

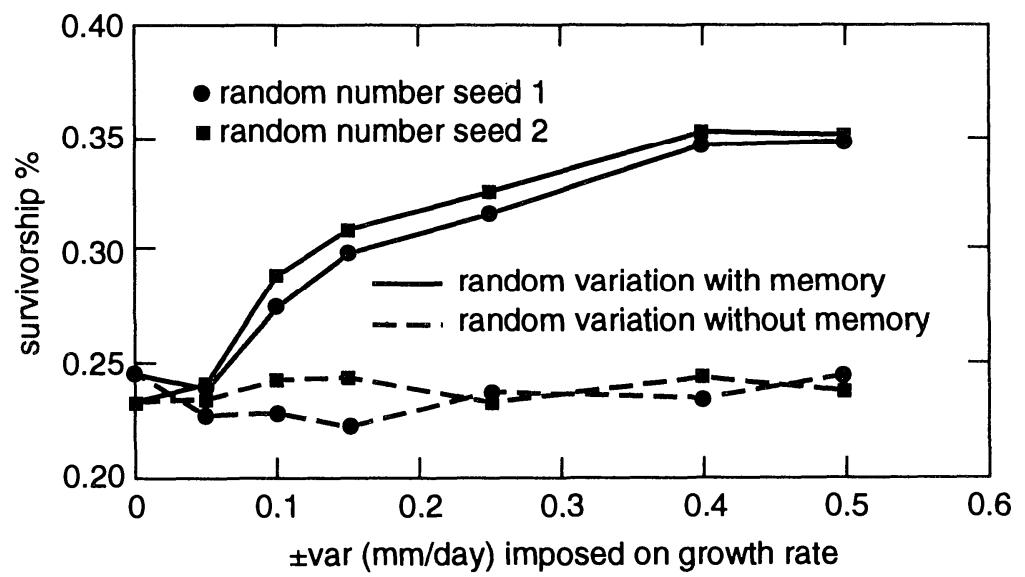

FIG. 4.-Case 1 percentage survival of fish after $60 \mathrm{~d}$ as a function of variability in daily growth with memory and without memory.

These results indicate the need for a much better understanding of the limits on the maximum daily growth of individual fish through ontogeny, as such limits can have a large effect on the consequences of variability in daily growth with memory. The above comparisons also underscore the importance of memory in growth rate. No studies have examined variations in daily growth rates in individually marked larval fish. Such research would prove useful. Without this correlation of variance in growth rates from $1 \mathrm{~d}$ to the next, the variance itself has little effect. 


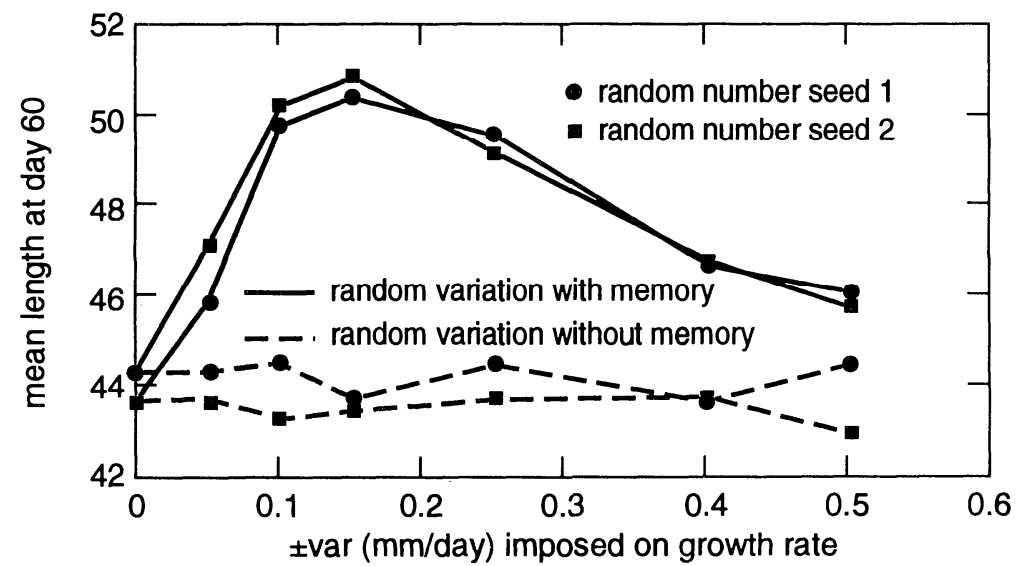

FIG. 5.-Case 1 mean length after $60 \mathrm{~d}$ as a function of variability in daily growth with memory and without memory.

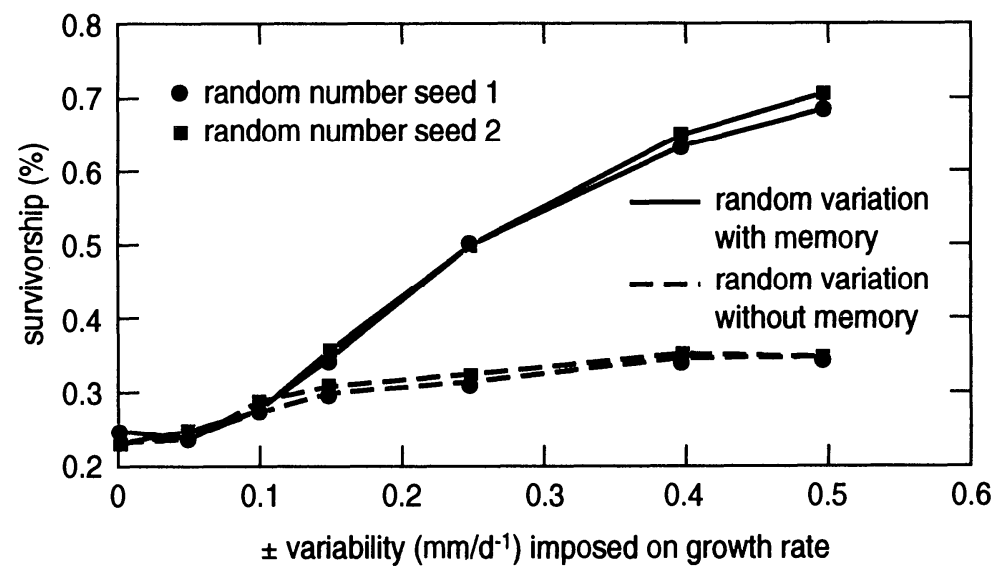

FIG. 6.- Case 1 comparisons of survival after $60 \mathrm{~d}$ as a function of variability in daily growth with memory for limits on maximum daily growth rate of 1.0 and $1.5 \mathrm{~mm} / \mathrm{d}$.

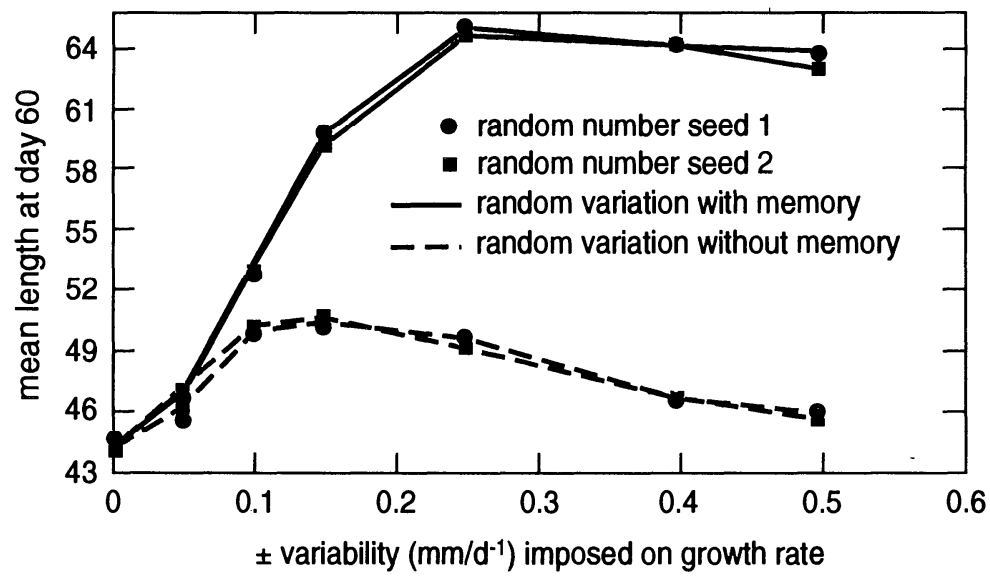

FIG. 7.-Case 1 comparisons of mean length after $60 \mathrm{~d}$ as a function of variability in daily growth with memory for limits on maximum daily growth rate of 1.0 and $1.5 \mathrm{~mm} / \mathrm{d}$. 
Case 2.

The comparison between the partial differential equation solution (10) and the equivalent Monte Carlo simulation is shown in figure 8. The specific values for the parameters of the models are shown in table 1 . The cohort size-frequency distribution is shown for three times, $t=$ 20, 40, and $60 \mathrm{~d}$. Agreement is acceptably close, though the simulation values again tend to fall slightly below the partial differential equation values. The absence of skewing to the right of the size-frequency distributions in figure 8 may be surprising, as such skewing is frequently observed in fish populations. However, even though the growth rate in this case increases with size, it can be demonstrated mathematically that an initially normal distribution of sizes will remain normal through time. Other mechanisms, such as an unequal distribution of allocation of limiting resources among individuals, if included in the model, could produce skewed sizefrequency distributions through time. It has been shown that when the food availability to smaller fish becomes more limiting than food availability to larger fish, skewing and even the emergence of size bimodality can occur (DeAngelis and Coutant 1982).

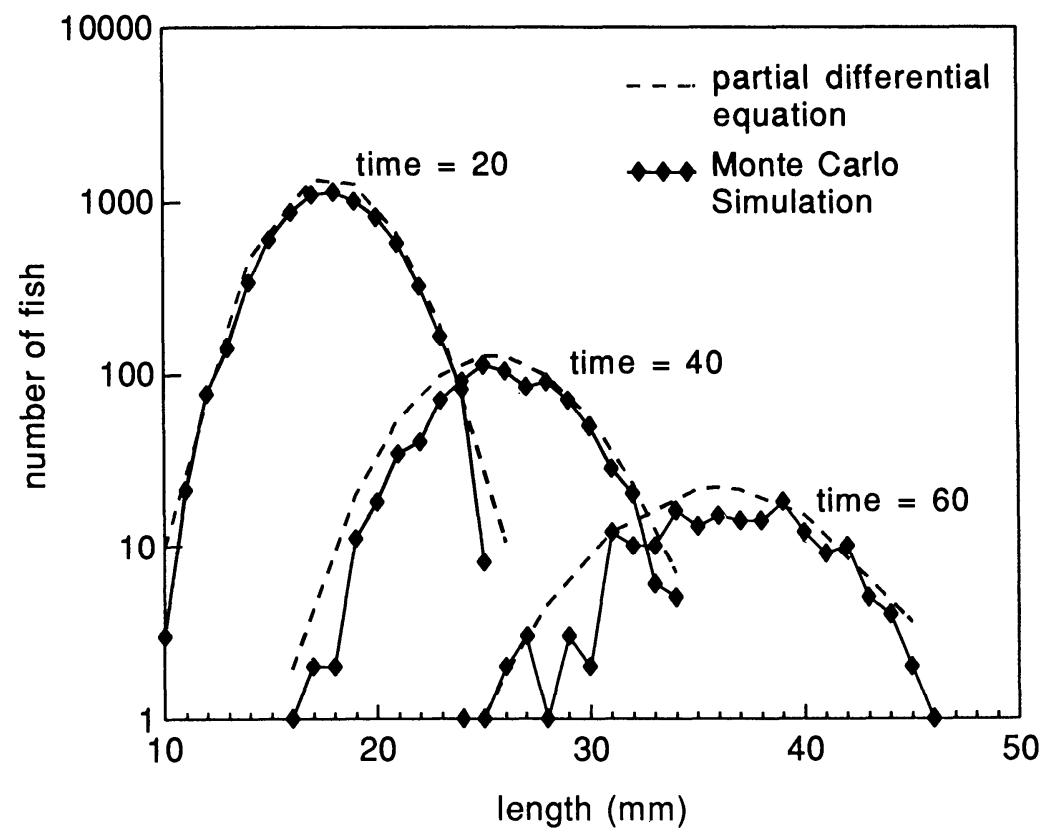

FIG. 8.-Case 2 comparisons between the partial differential equation solution, eq. (10) (dashed line), and the equivalent Monte Carlo simulation (solid line) for the size-frequency distribution of the cohort. An initial 200,000 fish were used in the Monte Carlo simulation.

As in case 1, the Monte Carlo simulation is next changed by allowing day-today changes in the growth increment by amounts lying randomly in the interval $\pm 0.15 \mathrm{~mm} / \mathrm{d}$ (with no memory). We anticipated in this case that there might be some interaction of the size-dependent growth rate with the stochastic variation that would allow some small fraction of the fish to attain very large growth rates and skew the distribution at $60 \mathrm{~d}$. However, there was no indication of any difference between the baseline and stochastic simulations (fig. 9). Hara (1984) has shown, in analyzing partial differential equation models of plant populations, however, that if the stochastic variation occurs not in the daily growth, $g_{1} S$, but in the relative growth rate, $g_{1} S / S=g_{1}$, there can be a much more dramatic effect on the size-frequency distribution through time. 


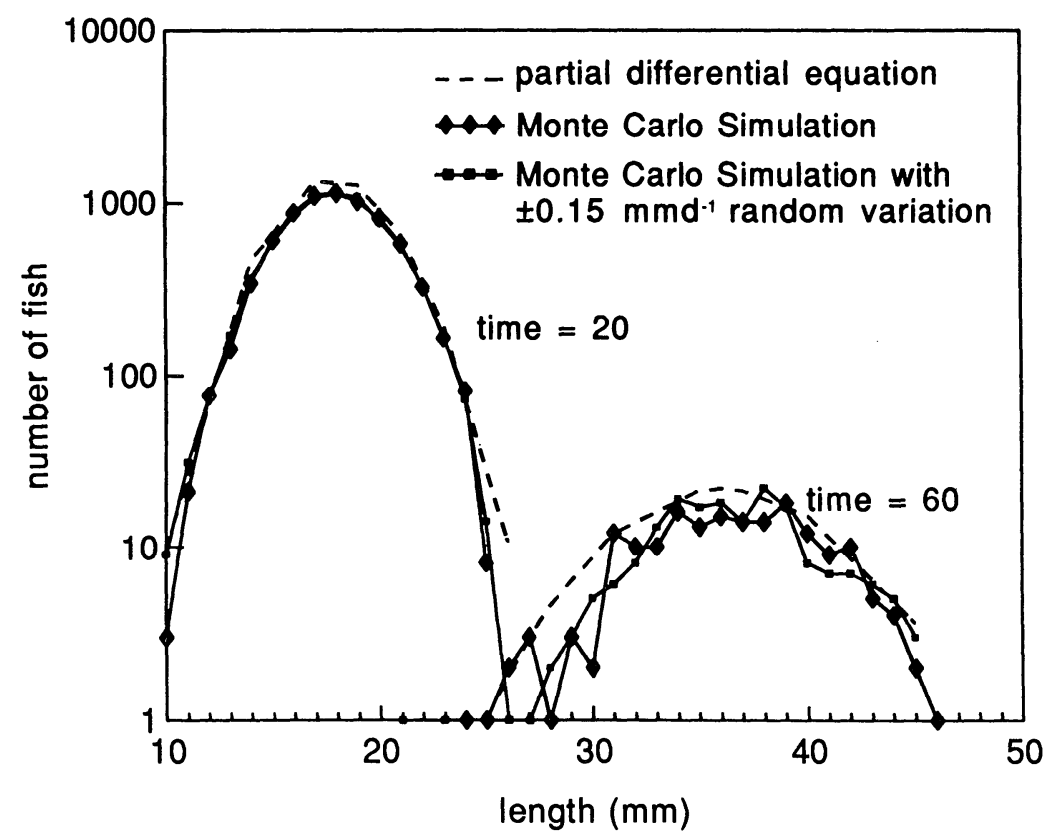

FIG. 9.-Case 2 size-frequency distributions from individual-based computer simulations of a fish cohort after 20 and $60 \mathrm{~d}$. The diamonds represent the case in which there was no stochasticity in daily growth, whereas the squares represent daily random variation in growth increment of up to $\pm 0.15 \mathrm{~mm} / \mathrm{d}$.

Case 3.

The comparison between the partial differential equation model (13) and the equivalent Monte Carlo simulation for the cohort size-frequency distribution is shown in figure 10. The specific parameter values for the model are shown in table 1. The cohort size-frequency distributions are compared for two times, $t=40$ and $60 \mathrm{~d}$. Agreement is once again very good. (Although a maximum size of $50 \mathrm{~mm}$ may be unrealistically small for a fish that hatches at 12 $\mathrm{mm}$, the main purpose of the simulation was to compare the results of methods, an objective that is not affected by a somewhat unrealistic choice of a parameter.)

As before, one can easily add special features to the Monte Carlo simulation. One option is to make the growth rate density-dependent. For example, the limitation of size to $S_{\max }=50$ might be relaxed as number of fish in the cohort decline. This situation can be simulated by replacing $S_{\max }$ with $S_{\max }\left(N_{0} / N\right)$, where $N_{0} / N$ is the ratio of the initial number of fish to the current number. As $N$ decreases through mortality, the upper limit on size becomes progressively larger. The simulation results for this case show the size-frequency distribution at 40 and especially $60 \mathrm{~d}$ to be shifted considerably to the right of the baseline cases (fig. 11). Adding stochasticity (in the range $\pm 0.15 \mathrm{~mm} / \mathrm{d}$ ) to the growth rate with no memory results in little change to this explicitly density-dependent case (fig. 11). Although equation (13) could not be solved analytically in the density-dependent case, it could be solved numerically. This was done by an approximation method, using the analytical solution (13) to step the solution one day at a time and adjusting $S_{\max }$ by the ratio $N_{0} / N$ on each time step. The numerical solution is shown for $t=60 \mathrm{~d}$ in figure 11 , and its agreement with the Monte Carlo simulation is good enough for many of the purposes to which such models would be put. 


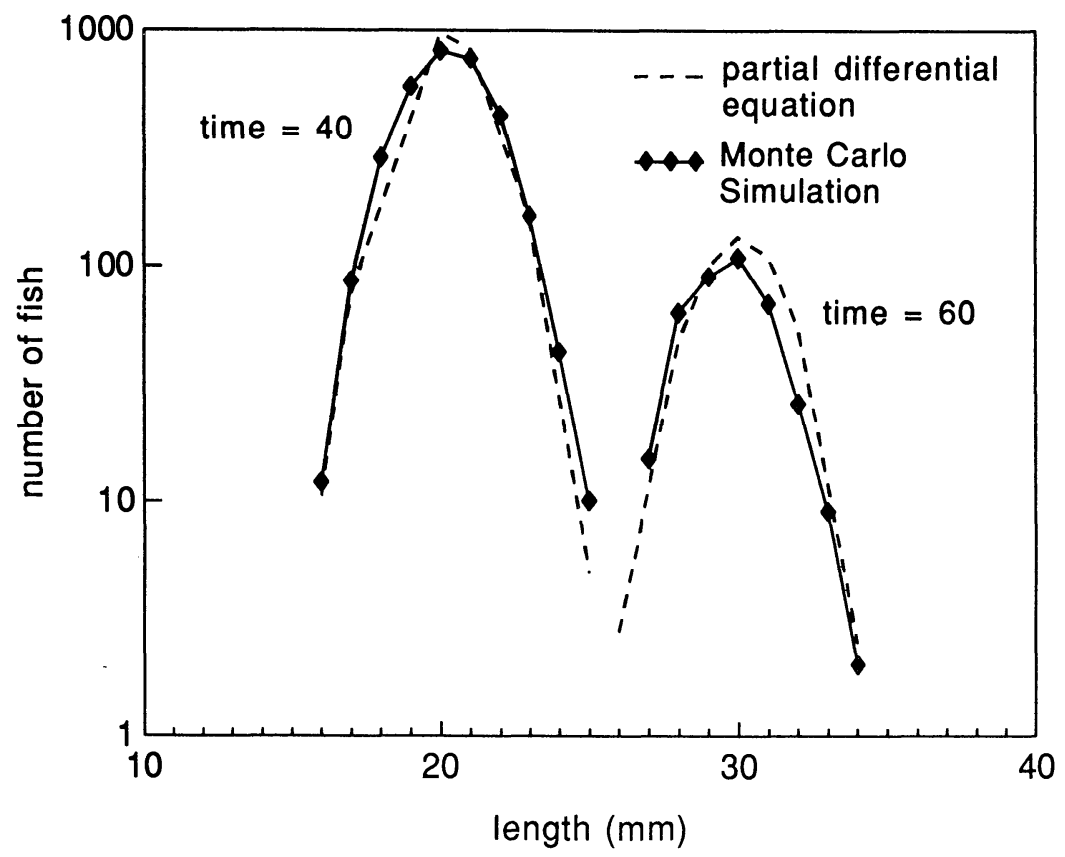

FIG. 10.-Case 3 comparisons between the partial differential equation solution, eq. (13) (dashed line), and the equivalent Monte Carlo simulation (solid line) for the size-frequency distribution of the cohort. An initial 20,000 fish were used in the Monte Carlo simulation.

\section{DISCUSSION}

The results of this work provide some useful insights concerning the application of modeling to the study of recruitment variability in fish populations. They show that equivalent partial differential equation models and Monte Carlo models of fish cohorts can produce very similar results for the limiting cases in which analytical solutions are possible. The results do have slight systematic differences because partial differential equations are continuous-time models, whereas the Monte Carlo simulations work on discrete daily time steps. Survival is lower in the daily compounded mortality. This did not make much difference in the cases considered, but for higher precision in the Monte Carlo simulations, one might want to consider taking smaller time steps (e.g., fraction of a day).

The cases considered are simple ones, and the results do not prove that the Monte Carlo simulation approach will yield precise approximations of the partial differential equations in all cases. However, in most modeling studies the uncertainty in the parameter values is great enough anyway that very high precision in model analysis is not crucial. If the level of agreement shown in the comparisons of the two approaches is acceptable for typical problems of computing size distributions in populations, then it means that Monte Carlo simulations with as few as 20,000 individual organisms can provide a good description of the changing size-frequency distribution. This appears to hold fairly well even when mortality is high, so that numbers become quite low by the end of the simulation.

The addition of stochasticity without memory in the Monte Carlo model produced results that did not deviate significantly from the baseline cases. This may imply that the partial differential equation approach may often apply even when there is some stochasticity in the system. However, from Hara's (1984) model results of stochasticity in growth in plant populations, this may not be generalizable, particularly to situations in which the stochasticity in 
question occurs in a rate coefficient for exponential growth.

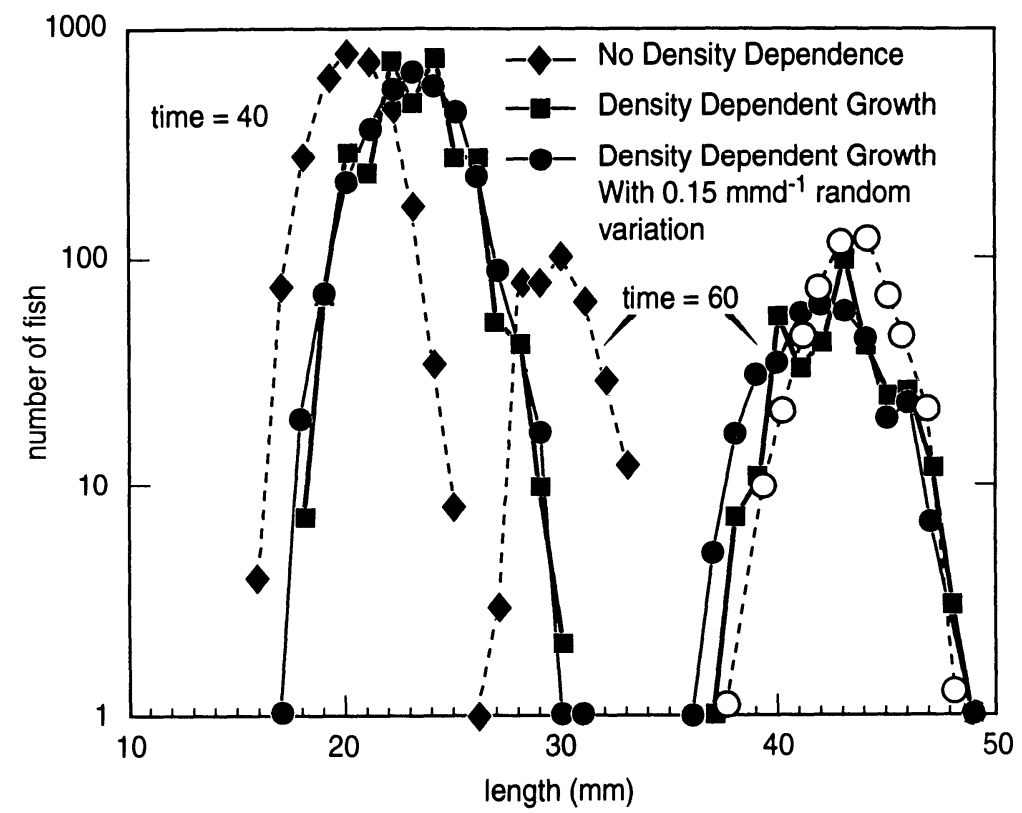

FIG. 11.-Case 3 size-frequency distributions from individual-based computer simulations of a fish cohort after 40 and $60 \mathrm{~d}$. The dotted lines represent the simulation in which there are no positive effects on growth rate as number density decreases. The thick solid lines represent the simulation in which the upper limit on size increases as $N / N_{0}$ decreases. The thin solid lines represent the simulation in which stochasticity in daily growth $( \pm 0.15 \mathrm{~mm} / \mathrm{d})$ is added to the density-dependent effect. The open circles at $t=60$ represent a numerical solution of the partial differential eq. (13).

It was also shown that more complex formulations of individual growth, such as daily growth rates that are correlated, can cause dramatic changes in the dynamics of the cohort. Although the results of these simulations cannot be checked against simple partial differential equation models, the fact that we have checked other cases against the partial differential equation models gives us confidence that the results are correct. Additional biological research on correlation of daily growth rates of individual larvae would help in forecasting the elaboration of variance in cohort size structure.

This work also showed the effects of other assumptions. The assumption concerning the ontogeny of maximum daily growth rate among individuals had a effect on the way in which variability in growth rate with memory could affect the survival and mean length of fish after 60 d. Our assumptions are realistic relative to observed average growth rates of marine larvae (Pepin 1991), but again, we know little regarding variation among and within individual growth rates. Knowledge of this maximum daily growth rate is critical to predictions of fish population behavior in variable environments. Recruitment in fishes seems to depend strongly on individual variation. Models such as those presented here suggest where individual variation in parameters could have the greatest effect on cohort survival and size structure. In this way they help clarify research priorities for biologists interested in recruitment.

\section{ACKNOWLEDGMENTS}

This research was sponsored in part by the Electric Power Research Institute under contract no. RP2932-2 (DOE no. ERD-87-672) with the U.S. Department of Energy under 
contract no. DE-AC05-84OR21400 with Martin Marietta Energy Systems, and in part by grant no. NAI6RG0492-01 from the Coastal Ocean Program of the National Oceanic and Atmospheric Administration (NOAA) to the University of North Carolina Sea Grant College Program. The views expressed herein are those of the author(s) and do not reflect the views of NOAA or any of its subagencies.

\section{LITERATURE CITED}

Caswell, H. 1989. Matrix population models: construction, analysis, and interpretation. Sinauer, Sunderland, Mass.

Caswell, H., and A. M. John. 1992. From the individual to the population in demographic models. Pages 36-61 in D. L. DeAngelis and L. J. Gross, eds. Individual-based models and approaches in ecology. Chapman \& Hall, New York.

DeAngelis, D. L., and C. C. Coutant. 1982. Genesis of bimodal size distributions in species cohorts. Transactions of the American Fisheries Society 111:384-388.

DeAngelis, D. L., and M. A. Huston. 1987. Effects of growth rates in models of size distribution formation in plants and animals. Ecological Modelling 36:119-137.

DeAngelis, D. L., and J. S. Mattice. 1980. Implications of a partial differential equation cohort model. Mathematical Biosciences 47:271-285.

DeAngelis, D. L., and K. A. Rose. 1992. Which individual-based approach is most appropriate for a given problem? Pages 67-87 in D. L. DeAngelis and L. J. Gross, eds. Individualbased models and approaches in ecology. Chapman \& Hall, New York.

DeAngelis, D. L., P. A. Hackney, and J. C. Webb. 1980. A partial differential equation model of changing sizes and numbers in a cohort of juvenile fish. Environmental Biology of Fishes 5:261-266.

Ebenman, B., and L. Persson, eds. 1988. Size-structured populations: ecology and evolution. Springer, Berlin.

Hara, T. 1984. A stochastic model and the moment dynamics of the growth and size distribution in plant populations. Journal of Theoretical Biology 109:173-190.

Houde, E. D. 1987. Fish early life history dynamics and recruitment variability. American Fisheries Society Symposium 2:17-29.

-------- 1989. Comparative growth, mortality, and energetics of marine fish larvae: temperature and implied latitudinal effect. Fisheries Bulletin 87:471-479.

Hunter, J. R. 1981. Feeding ecology and predation of marine fish larvae. Pages 33-79 in R. Lasker, ed. Marine fish larvae. Washington Sea Grant, University of Washington Press, Seattle.

Huston, M. A., D. L. DeAngelis, and W. M. Post. 1988. New computer models unify ecological theory. BioScience 38:682-691.

Łomnicki, A. 1988. Population ecology of individuals. Princeton University Press, Princeton, N.J.

Metz, J. A. J., and O. Diekmann, eds. 1986. The dynamics of physiologically structured populations. Springer Lecture Notes in Biomathematics. Vol. 68. Springer, Berlin.

Metz, J. A. J., A. M. de Roos, and F. van den Bosch. 1988. Population models incorporating physiological structure: a quick survey of the basic concepts and an application to sizestructured population dynamics in waterfleas. Pages 106-126 in B. Ebenman and L. Persson, eds. Size-structured populations: ecology and evolution. Springer, Berlin. 
Miller, T. J., L. B. Crowder, J. A. Rice, and E. A. Marschall. 1988. Larval size and recruitment mechanisms in fishes: toward a conceptual framework. Canadian Journal of Fisheries and Aquatic Sciences 45:1657-1670.

Nisbet, R. M., and W. S. Gurney. 1982. Modeling fluctuating populations. Wiley, London.

Pepin, P. 1991. Effect of temperature and size on development, mortality, and survival rates of the pelagic early life history stages of marine fish. Canadian Journal of Fisheries and Aquatic Sciences 48:503-518.

Persson, L. 1987. The effects of resource availability and distribution on size class interactions in perch Perca fluviatilis. Oikos 48:148-160.

Rice, J. A., L. B. Crowder, and F. P. Binkowski. 1985. Evaluating otolith analysis for bloater (Coregonus hoyi): do otoliths ring true? Transactions of the American Fisheries Society 114:532-539.

Rice, J. A., L. B. Crowder, and M. E. Holey. 1987. Exploration of mechanisms regulating larval survival in Lake Michigan bloater: a recruitment analysis based on characteristics of individual larvae. Transactions of the American Fisheries Society 116:703-719.

Rice, J. A., T. J. Miller, L. B. Crowder, E. A. Marschall, A. S. Trebitz, and D. L. DeAngelis. 1993. Growth rate variation and larval survival: inferences from an individual-based, size-dependent predation model. Canadian Journal of Fisheries and Aquatic Sciences 50:133-142.

Sinclair, M. 1988. Marine populations: an essay on population regulation and speciation. Washington Sea Grant, University of Washington Press, Seattle.

Sinko, J. W., and W. Streifer. 1967. A new model for age-size structure of a population. Ecology 48:910-918.

Van Sickle, J. 1977. Analysis of a distributed-parameter population model based on physiological age. Journal of Theoretical Biology 64:571-586.

Wilbur, H. M. 1988. Interactions between growing predators and growing prey. Pages 157-172 in B. Ebenman and L. Persson, eds. Size-structured populations: ecology and evolution. Springer, Berlin.

Wismer, D. A., D. L. DeAngelis, and B. J. Shuter. 1985. An empirical model of size distributions of smallmouth bass. Transactions of the American Fisheries Society 114:737-742. 